\title{
Identified Particles in Quark and Gluon Jets
}

DELPHI Collaboration

\begin{abstract}
A sample of about 1.4 million hadronic $Z$ decays, selected among the data recorded by the DELPHI detector at LEP during 1994, was used to measure for the first time the momentum spectra of $K^{+}, K^{0}, p, \Lambda$ and their antiparticles in gluon and quark jets. As observed for inclusive charged particles, the production spectra of identified particles were found to be softer in gluon jets than in quark jets, with a higher total multiplicity.
\end{abstract}


P.Abreu ${ }^{21}$, W.Adam ${ }^{50}$, T.Adye ${ }^{37}$, I.Ajinenko ${ }^{42}$, G.D.Alekseev ${ }^{16}$, R.Alemany ${ }^{49}$, P.P.Allport ${ }^{22}$, S.Almehed ${ }^{24}$, U.Amaldi $^{9}, \quad$ S.Amato ${ }^{47}, \quad$ A.Andreazza ${ }^{28}$, M.L.Andrieux ${ }^{14}$, P.Antilogus ${ }^{9}, \quad$ W-D.Apel ${ }^{17}, \quad$ B.Asman ${ }^{44}$, J-E.Augustin ${ }^{25}$, A.Augustinus ${ }^{9}$, P.Baillon ${ }^{9}$, P.Bambade ${ }^{19}$, F.Barao $^{21}$, M.Barbi $^{47}$, G.Barbiellini ${ }^{46}$, D.Y.Bardin ${ }^{16}$, G.Barker $^{9}$, A.Baroncelli ${ }^{40}$, O.Barring ${ }^{24}$, J.A.Barrio ${ }^{26}$, W.Bartl ${ }^{50}$, M.J.Bates ${ }^{37}$, M.Battaglia ${ }^{15}$, M.Baubillier ${ }^{23}$, J.Baudot $^{39}$, K-H.Becks ${ }^{52}$, M.Begalli ${ }^{6}$, P.Beilliere ${ }^{8}$, Yu.Belokopytov ${ }^{9,53}$, A.C.Benvenuti ${ }^{5}$, M.Berggren ${ }^{47}$, D.Bertini ${ }^{25}$, D.Bertrand ${ }^{2}$, M.Besancon ${ }^{39}$, F.Bianchi ${ }^{45}$, M.Bigi ${ }^{45}$, M.S.Bilenky ${ }^{16}$, P.Billoir ${ }^{23}$, M-A.Bizouard ${ }^{19}$, D.Bloch $^{10}$, M.Blume ${ }^{52}$, T.Bolognese ${ }^{39}$, M.Bonesini ${ }^{28}$, W.Bonivento ${ }^{28}$, P.S.L.Booth ${ }^{22}$, C.Bosio ${ }^{40}$, O.Botner $^{48}$, E.Boudinov $^{31}$, B.Bouquet ${ }^{19}$, C.Bourdarios ${ }^{9}$, T.J.V.Bowcock ${ }^{22}$, M.Bozzo ${ }^{13}$, P.Branchini ${ }^{40}$, K.D.Brand ${ }^{36}$, T.Brenke $^{52}$, R.A.Brenner ${ }^{15}$, C.Bricman ${ }^{2}$, R.C.A.Brown ${ }^{9}$, P.Bruckman ${ }^{18}$, J-M.Brunet ${ }^{8}$, L.Bugge ${ }^{33}$, T.Buran ${ }^{33}$, T.Burgsmueller ${ }^{52}$, P.Buschmann ${ }^{52}$, S.Cabrera ${ }^{49}$, M.Caccia $^{28}$, M.Calvi ${ }^{28}$, A.J.Camacho Rozas ${ }^{41}$, T.Camporesi $^{9}$, V.Canale ${ }^{38}$, M.Canepa $^{13}$, K.Cankocak ${ }^{44}$,F.Cao ${ }^{2}$,F.Carena ${ }^{9}$, L.Carroll ${ }^{22}$, C.Caso ${ }^{13}$, M.V.Castillo Gimenez ${ }^{49}$, A.Cattai $^{9}$, F.R.Cavallo ${ }^{5}$, V.Chabaud ${ }^{9}$, Ph.Charpentier ${ }^{9}$, L.Chaussard ${ }^{25}$, P.Checchia ${ }^{36}$, G.A.Chelkov ${ }^{16}$, M.Chen $^{2}$, R.Chierici ${ }^{45}$, P.Chliapnikov ${ }^{42}$, P.Chochula ${ }^{7}$, V.Chorowicz ${ }^{9}$, J.Chudoba ${ }^{30}$, V.Cindro ${ }^{43}$, P.Collins ${ }^{9}$, R.Contri ${ }^{13}$, E.Cortina ${ }^{49}$, G.Cosme ${ }^{19}$, F.Cossutti ${ }^{46}$, J-H.Cowell ${ }^{22}$, H.B.Crawley ${ }^{1}$, D.Crennell ${ }^{37}$, G.Crosetti $^{13}$, J.Cuevas Maestro $^{34}$, S.Czellar ${ }^{15}$, E.Dahl-Jensen ${ }^{29}$, J.Dahm ${ }^{52}$, B.Dalmagne ${ }^{19}$, M.Dam ${ }^{29}$, G.Damgaard ${ }^{29}$, P.D.Dauncey ${ }^{37}$, M.Davenport ${ }^{9}$, W.Da Silva ${ }^{23}$, C.Defoix ${ }^{8}$, A.Deghorain ${ }^{2}$, G.Della Ricca ${ }^{46}$, P.Delpierre ${ }^{27}$, N.Demaria $^{35}$, A.De Angelis ${ }^{9}$, W.De Boer ${ }^{17}$, S.De Brabandere ${ }^{2}$, C.De Clercq ${ }^{2}$, C.De La Vaissiere ${ }^{23}$, B.De Lotto ${ }^{46}$, A.De Min ${ }^{36}$, L.De Paula ${ }^{47}$, C.De Saint-Jean ${ }^{39}$, H.Dijkstra ${ }^{9}$, L.Di Ciaccio ${ }^{38}$, A.Di Diodato ${ }^{38}$, F.Djama $^{10}$, A.Djannati ${ }^{8}$, J.Dolbeau ${ }^{8}$, K.Doroba ${ }^{51}$, M.Dracos $^{10}$, J.Drees ${ }^{52}$, K.-A.Drees ${ }^{52}$, M.Dris ${ }^{32}$, J-D.Durand $^{25,9}$, D.Edsall ${ }^{1}$, R.Ehret ${ }^{17}$, G.Eigen ${ }^{4}$, T.Ekelof ${ }^{48}$, G.Ekspong ${ }^{44}$, M.Elsing ${ }^{9}$, J-P.Engel ${ }^{10}$, B.Erzen ${ }^{43}$, M.Espirito Santo ${ }^{21}$, E.Falk ${ }^{24}$, D.Fassouliotis ${ }^{32}$, M.Feindt ${ }^{9}$, A.Ferrer ${ }^{49}$, S.Fichet ${ }^{23}$, T.A.Filippas ${ }^{32}$, A.Firestone ${ }^{1}$, P.-A.Fischer ${ }^{10}$, H.Foeth ${ }^{9}$, E.Fokitis ${ }^{32}$, F.Fontanelli ${ }^{13}$, F.Formenti ${ }^{9}$, B.Franek ${ }^{37}$, P.Frenkiel ${ }^{8}$, D.C.Fries ${ }^{17}$, A.G.Frodesen ${ }^{4}$, R.Fruhwirth ${ }^{50}$, F.Fulda-Quenzer ${ }^{19}$, J.Fuster ${ }^{49}$, A.Galloni ${ }^{22}$, D.Gamba ${ }^{45}$, M.Gandelman $^{47}$, C.Garcia $^{49}$, J.Garcia ${ }^{41}$, C.Gaspar ${ }^{9}$, U.Gasparini ${ }^{36}$, Ph.Gavillet ${ }^{9}$, E.N.Gazis ${ }^{32}$, D.Gele ${ }^{10}$, J-P.Gerber $^{10}$, L.Gerdyukov $^{42}$, R.Gokieli ${ }^{51}$, B.Golob ${ }^{43}$, G.Gopal ${ }^{37}$, L.Gorn ${ }^{1}$, M.Gorski $^{51}$, Yu.Gouz ${ }^{45,53}$, V.Gracco ${ }^{13}$, E.Graziani ${ }^{40}$, C.Green ${ }^{22}$, A.Grefrath ${ }^{52}$, P.Gris ${ }^{39}$, G.Grosdidier ${ }^{19}$, K.Grzelak ${ }^{51}$, S.Gumenyuk ${ }^{28,53}$, P.Gunnarsson $^{44}$, M.Gunther ${ }^{48}$, J.Guy ${ }^{37}$, F.Hahn ${ }^{9}$, S.Hahn ${ }^{52}$, Z.Hajduk ${ }^{18}$, A.Hallgren ${ }^{48}$, K.Hamacher ${ }^{52}$, F.J.Harris ${ }^{35}$, V.Hedberg ${ }^{24}$, R.Henriques ${ }^{21}$, J.J.Hernandez ${ }^{49}$, P.Herquet $^{2}$, H.Herr ${ }^{9}$, T.L.Hessing ${ }^{35}$, J.-M.Heuser $^{52}$, E.Higon ${ }^{49}$, H.J.Hilke ${ }^{9}$, T.S.Hill ${ }^{1}$, S-O.Holmgren ${ }^{44}$, P.J.Holt ${ }^{35}$, D.Holthuizen ${ }^{31}$, S.Hoorelbeke ${ }^{2}$, M.Houlden $^{22}$, J.Hrubec ${ }^{50}$, K.Huet ${ }^{2}$, K.Hultqvist ${ }^{44}$, J.N.Jackson ${ }^{22}$, R.Jacobsson ${ }^{44}$, P.Jalocha ${ }^{18}$, R.Janik ${ }^{7}$, Ch.Jarlskog $^{24}$, G.Jarlskog ${ }^{24}$, P.Jarry ${ }^{39}$, B.Jean-Marie ${ }^{19}$, E.K.Johansson ${ }^{44}$, L.Jonsson ${ }^{24}$, P.Jonsson ${ }^{24}$, C.Joram ${ }^{9}$, P.Juillot ${ }^{10}$, M.Kaiser ${ }^{17}$, F.Kapusta ${ }^{23}$, K.Karafasoulis ${ }^{11}$, M.Karlsson ${ }^{44}$, E.Karvelas ${ }^{11}$, A.Katargin ${ }^{42}$, S.Katsanevas $^{3}$, E.C.Katsoufis ${ }^{32}$, R.Keranen ${ }^{4}$, Yu.Khokhlov ${ }^{42}$, B.A.Khomenko ${ }^{16}$, N.N.Khovanski ${ }^{16}$, B.King ${ }^{22}$, N.J.Kjaer ${ }^{31}$, O.Klapp ${ }^{52}$, H.Klein ${ }^{9}$, A.Klovning ${ }^{4}$, P.Kluit ${ }^{31}$, B.Koene ${ }^{31}$, P.Kokkinias ${ }^{11}$, M.Koratzinos $^{9}$, K.Korcyl $^{18}$, V.Kostioukhine ${ }^{42}$, C.Kourkoumelis ${ }^{3}$, O.Kouznetsov ${ }^{13,16}{ }^{\text {, M.Krammer }}{ }^{50}$, C.Kreuter $^{9}$, I.Kronkvist ${ }^{24}$, Z.Krumstein $^{16}$, W.Krupinski ${ }^{18}$, P.Kubinec ${ }^{7}$, W.Kucewicz ${ }^{18}$, K.Kurvinen ${ }^{15}$, C.Lacasta ${ }^{49}$, I.Laktineh ${ }^{25}$, J.W.Lamsa $^{1}$, L.Lanceri ${ }^{46}$, D.W.Lane ${ }^{1}$, P.Langefeld ${ }^{52}$, V.Lapin ${ }^{42}$, J-P.Laugier ${ }^{39}$, R.Lauhakangas ${ }^{15}$, G.Leder $^{50}$, F.Ledroit $^{14}$, V.Lefebure ${ }^{2}$, C.K.Legan ${ }^{1}$, R.Leitner ${ }^{30}$, J.Lemonne ${ }^{2}$, G.Lenzen ${ }^{52}$, V.Lepeltier ${ }^{19}$, T.Lesiak ${ }^{18}$, J.Libby $^{35}$, D.Liko ${ }^{9}$, R.Lindner ${ }^{52}$, A.Lipniacka ${ }^{44}$, I.Lippi ${ }^{36}$, B.Loerstad ${ }^{24}$, J.G.Loken ${ }^{35}$, J.M.Lopez ${ }^{41}$, D.Loukas $^{11}$, P.Lutz ${ }^{39}$, L.Lyons ${ }^{35}$, J.MacNaughton ${ }^{50}$, G.Maehlum ${ }^{17}$, J.R.Mahon ${ }^{6}$, T.G.M.Malmgren ${ }^{44}$, V.Malychev $^{16}$, F.Mandl ${ }^{50}$, J.Marco ${ }^{41}$, R.Marco ${ }^{41}$, B.Marechal ${ }^{47}$, M.Margoni ${ }^{36}$, J-C.Marin ${ }^{9}$, C.Mariotti ${ }^{9}$, A.Markou ${ }^{11}$, C.Martinez-Rivero ${ }^{34}$, F.Martinez-Vidal ${ }^{49}$, S.Marti i Garcia ${ }^{22}$, J.Masik ${ }^{30}$, F.Matorras ${ }^{41}$, C.Matteuzzi $^{28}$, G.Matthiae ${ }^{38}$, M.Mazzucato ${ }^{36}$, M.Mc Cubbin ${ }^{22}$, R.Mc Kay ${ }^{1}$, R.Mc Nulty ${ }^{22}$, J.Medbo ${ }^{48}$, M.Merk $^{31}$, C.Meroni $^{28}$,S.Meyer $^{17}$,W.T.Meyer ${ }^{1}$, M.Michelotto ${ }^{36}$, E.Migliore $^{45}$, L.Mirabito ${ }^{25}$, W.A.Mitaroff ${ }^{50}$, U.Mjoernmark $^{24}$, T.Moa ${ }^{44}$, R.Moeller ${ }^{29}$, K.Moenig ${ }^{9}$, M.R.Monge ${ }^{13}$, P.Morettini ${ }^{13}$, H.Mueller ${ }^{17}$, K.Muenich $^{52}$, M.Mulders $^{31}$, L.M.Mundim ${ }^{6}$, W.J.Murray ${ }^{37}$, B.Muryn ${ }^{14,18}$, G.Myatt ${ }^{35}$, F.Naraghi ${ }^{14}$, F.L.Navarria ${ }^{5}$, S.Navas ${ }^{49}$, K.Nawrocki ${ }^{51}$, P.Negri ${ }^{28}$, W.Neumann ${ }^{52}$, N.Neumeister ${ }^{50}$, R.Nicolaidou ${ }^{3}$, B.S.Nielsen ${ }^{29}$, M.Nieuwenhuizen ${ }^{31}$, V.Nikolaenko ${ }^{10}$, P.Niss ${ }^{44}$, A.Nomerotski ${ }^{36}$, A.Normand ${ }^{35}$, M.Novak ${ }^{12}$, W.Oberschulte-Beckmann ${ }^{17}$, V.Obraztsov ${ }^{42}$, A.G.Olshevski ${ }^{16}$, A.Onofre ${ }^{21}$, R.Orava ${ }^{15}$, K.Osterberg ${ }^{15}$, A.Ouraou ${ }^{39}$, P.Paganini ${ }^{19}$, M.Paganoni ${ }^{9,28}$, P.Pages ${ }^{10}$, R.Pain ${ }^{23}$, H.Palka ${ }^{18}$, Th.D.Papadopoulou ${ }^{32}$, K.Papageorgiou ${ }^{11}$, L.Pape ${ }^{9}$, C.Parkes $^{35}$, F.Parodi ${ }^{13}$, A.Passeri ${ }^{40}$, M.Pegoraro ${ }^{36}$, L.Peralta ${ }^{21}$, M.Pernicka ${ }^{50}$, A.Perrotta ${ }^{5}$, C.Petridou ${ }^{46}$, A.Petrolini $^{13}$, M.Petrovyck ${ }^{42}$, H.T.Phillips ${ }^{37}$, G.Piana ${ }^{13}$, F.Pierre ${ }^{39}$, M.Pimenta ${ }^{21}$, T.Podobnik ${ }^{43}$, O.Podobrin ${ }^{9}$, M.E.Pol ${ }^{6}$, G.Polok ${ }^{18}$, P.Poropat ${ }^{46}$, V.Pozdniakov ${ }^{16}$, P.Privitera ${ }^{38}$, N.Pukhaeva ${ }^{16}$, A.Pullia ${ }^{28}$, D.Radojicic ${ }^{35}$, S.Ragazzi $^{28}$, H.Rahmani ${ }^{32}$, J.Rames ${ }^{12}$, P.N.Ratoff ${ }^{20}$, A.L.Read ${ }^{33}$, M.Reale ${ }^{52}$, P.Rebecchi ${ }^{19}$, N.G.Redaelli ${ }^{28}$, M.Regler ${ }^{50}$, D.Reid ${ }^{9}$, R.Reinhardt ${ }^{52}$, P.B.Renton ${ }^{35}$, L.K.Resvanis ${ }^{3}$, F.Richard ${ }^{19}$, J.Richardson ${ }^{22}$, J.Ridky ${ }^{12}$, G.Rinaudo $^{45}$, I.Ripp ${ }^{39}$, A.Romero ${ }^{45}$, I.Roncagliolo ${ }^{13}$, P.Ronchese ${ }^{36}$, L.Roos ${ }^{23}$, E.I.Rosenberg ${ }^{1}$, P.Roudeau ${ }^{19}$, T.Rovelli $^{5}$, W.Ruckstuhl ${ }^{31}$, V.Ruhlmann-Kleider ${ }^{39}$, A.Ruiz ${ }^{41}$, K.Rybicki ${ }^{18}$, A.Rybin ${ }^{42}$, H.Saarikko ${ }^{15}$, Y.Sacquin ${ }^{39}$, A.Sadovsky ${ }^{16}$, O.Sahr ${ }^{14}$, G.Sajot ${ }^{14}$, J.Salt ${ }^{49}$, J.Sanchez ${ }^{26}$, M.Sannino ${ }^{13}$, M.Schimmelpfennig ${ }^{17}$, H.Schneider ${ }^{17}$, U.Schwickerath ${ }^{17}$, M.A.E.Schyns ${ }^{52}$, G.Sciolla ${ }^{45}$, F.Scuri $^{46}$, P.Seager ${ }^{20}$, Y.Sedykh ${ }^{16}$, A.M.Segar ${ }^{35}$, A.Seitz $^{17}$, R.Sekulin ${ }^{37}$, L.Serbelloni ${ }^{38}$, R.C.Shellard ${ }^{6}$, P.Siegrist ${ }^{39}$, R.Silvestre ${ }^{39}$, S.Simonetti ${ }^{39}$, F.Simonetto $^{36}$, A.N.Sisakian ${ }^{16}$, B.Sitar ${ }^{7}$, T.B.Skaali ${ }^{33}$, G.Smadja $^{25}$, N.Smirnov ${ }^{42}$, O.Smirnova ${ }^{24}$, G.R.Smith ${ }^{37}$, R.Sosnowski ${ }^{51}$, D.Souza-Santos ${ }^{6}$, T.Spassov ${ }^{21}$, E.Spiriti ${ }^{40}$, P.Sponholz ${ }^{52}$, S.Squarcia ${ }^{13}$, D.Stampfer ${ }^{9}$, C.Stanescu ${ }^{40}$, S.Stanic ${ }^{43}$, S.Stapnes $^{33}$, I.Stavitski ${ }^{36}$, K.Stevenson ${ }^{35}$, A.Stocchi ${ }^{19}$, J.Strauss ${ }^{50}$, R.Strub ${ }^{10}$, B.Stugu ${ }^{4}$, M.Szczekowski ${ }^{51}$, 
M.Szeptycka ${ }^{51}$, T.Tabarelli ${ }^{28}$, J.P.Tavernet ${ }^{23}$, O.Tchikilev ${ }^{42}$, J.Thomas ${ }^{35}$, A.Tilquin ${ }^{27}$, J.Timmermans ${ }^{31}$, L.G.Tkatchev ${ }^{16}$, T.Todorov ${ }^{10}$, S.Todorova ${ }^{10}$, D.Z.Toet ${ }^{31}$, A.Tomaradze ${ }^{2}$, B.Tome ${ }^{21}$, A.Tonazzo ${ }^{28}$, L.Tortora ${ }^{40}$, G.Transtromer $^{24}$, D.Treille ${ }^{9}$, G.Tristram ${ }^{8}$, A.Trombini ${ }^{19}$, C.Troncon ${ }^{28}$, A.Tsirou ${ }^{9}$, M-L.Turluer ${ }^{39}$, I.A.Tyapkin ${ }^{16}$, M.Tyndel $^{37}$, S.Tzamarias ${ }^{22}$, B. Ueberschaer ${ }^{52}$, O.Ullaland ${ }^{9}$, V.Uvarov ${ }^{42}$, G.Valenti ${ }^{5}$, E.Vallazza ${ }^{9}$, $\begin{array}{ll}\text { C.Vander Velde } & \text { G.W.Van Apeldoorn } \\ & \end{array}$, P.Van Dam ${ }^{31}$, W.K.Van Doninck ${ }^{2}$ J.Van Eldik ${ }^{31}$, A.Van Lysebetten ${ }^{2}$, N.Vassilopoulos ${ }^{35}$, G.Vegni ${ }^{28}$, L.Ventura ${ }^{36}$, W.Venus ${ }^{37}$, F.Verbeure ${ }^{2}$, M.Verlato $^{36}$, L.S.Vertogradov $^{16}$, D.Vilanova ${ }^{39}$, P.Vincent ${ }^{25}$, L.Vitale $^{46}$, E.Vlasov ${ }^{42}$, A.S.Vodopyanov ${ }^{16}$, V.Vrba $^{12}$, H.Wahlen ${ }^{52}$, C.Walck ${ }^{44}$, M.Weierstall ${ }^{52}$, P.Weilhammer ${ }^{9}$, C.Weiser ${ }^{17}$, A.M.Wetherell ${ }^{9}$, D.Wicke ${ }^{52}$, J.H.Wickens $^{2}$, M.Wielers ${ }^{17}$, G.R.Wilkinson ${ }^{9}$, W.S.C.Williams ${ }^{35}$, M.Winter ${ }^{10}$, M.Witek ${ }^{18}$, T.Wlodek ${ }^{19}$, K.Woschnagg $^{48}$, K.Yip ${ }^{35}$, O.Yushchenko ${ }^{42}$, F.Zach ${ }^{25}$, A.Zaitsev ${ }^{42}$, A.Zalewska ${ }^{9}$, P.Zalewski ${ }^{51}$, D.Zavrtanik ${ }^{43}$, E.Zevgolatakos ${ }^{11}$, N.I.Zimin ${ }^{16}$, M.Zito $^{39}$, D.Zontar ${ }^{43}$, G.C.Zucchelli ${ }^{44}$, G.Zumerle $^{36}$

\footnotetext{
${ }^{1}$ Department of Physics and Astronomy, Iowa State University, Ames IA 50011-3160, USA

${ }^{2}$ Physics Department, Univ. Instelling Antwerpen, Universiteitsplein 1, B-2610 Wilrijk, Belgium and IIHE, ULB-VUB, Pleinlaan 2, B-1050 Brussels, Belgium

and Faculté des Sciences, Univ. de l'Etat Mons, Av. Maistriau 19, B-7000 Mons, Belgium

${ }^{3}$ Physics Laboratory, University of Athens, Solonos Str. 104, GR-10680 Athens, Greece

${ }^{4}$ Department of Physics, University of Bergen, Allégaten 55, N-5007 Bergen, Norway

${ }^{5}$ Dipartimento di Fisica, Università di Bologna and INFN, Via Irnerio 46, I-40126 Bologna, Italy

${ }^{6}$ Centro Brasileiro de Pesquisas Físicas, rua Xavier Sigaud 150, RJ-22290 Rio de Janeiro, Brazil and Depto. de Física, Pont. Univ. Católica, C.P. 38071 RJ-22453 Rio de Janeiro, Brazil

and Inst. de Física, Univ. Estadual do Rio de Janeiro, rua São Francisco Xavier 524, Rio de Janeiro, Brazil

${ }^{7}$ Comenius University, Faculty of Mathematics and Physics, Mlynska Dolina, SK-84215 Bratislava, Slovakia

${ }^{8}$ Collège de France, Lab. de Physique Corpusculaire, IN2P3-CNRS, F-75231 Paris Cedex 05, France

${ }^{9} \mathrm{CERN}, \mathrm{CH}-1211$ Geneva 23, Switzerland

${ }^{10}$ Centre de Recherche Nucléaire, IN2P3 - CNRS/ULP - BP20, F-67037 Strasbourg Cedex, France

${ }^{11}$ Institute of Nuclear Physics, N.C.S.R. Demokritos, P.O. Box 60228, GR-15310 Athens, Greece

${ }^{12}$ FZU, Inst. of Physics of the C.A.S. High Energy Physics Division, Na Slovance 2, 180 40, Praha 8, Czech Republic

${ }^{13}$ Dipartimento di Fisica, Università di Genova and INFN, Via Dodecaneso 33, I-16146 Genova, Italy

${ }^{14}$ Institut des Sciences Nucléaires, IN2P3-CNRS, Université de Grenoble 1, F-38026 Grenoble Cedex, France

${ }^{15}$ Research Institute for High Energy Physics, SEFT, P.O. Box 9, FIN-00014 Helsinki, Finland

${ }^{16}$ Joint Institute for Nuclear Research, Dubna, Head Post Office, P.O. Box 79, 101000 Moscow, Russian Federation

${ }^{17}$ Institut für Experimentelle Kernphysik, Universität Karlsruhe, Postfach 6980, D-76128 Karlsruhe, Germany

${ }^{18}$ Institute of Nuclear Physics and University of Mining and Metalurgy, Ul. Kawiory 26a, PL-30055 Krakow, Poland

${ }^{19}$ Université de Paris-Sud, Lab. de l'Accélérateur Linéaire, IN2P3-CNRS, Bât. 200, F-91405 Orsay Cedex, France

${ }^{20}$ School of Physics and Chemistry, University of Lancaster, Lancaster LA1 4YB, UK

${ }^{21}$ LIP, IST, FCUL - Av. Elias Garcia, 14-1 ${ }^{\circ}$, P-1000 Lisboa Codex, Portugal

${ }^{22}$ Department of Physics, University of Liverpool, P.O. Box 147, Liverpool L69 3BX, UK

${ }^{23}$ LPNHE, IN2P3-CNRS, Universités Paris VI et VII, Tour 33 (RdC), 4 place Jussieu, F-75252 Paris Cedex 05, France

${ }^{24}$ Department of Physics, University of Lund, Sölvegatan 14, S-22363 Lund, Sweden

${ }^{25}$ Université Claude Bernard de Lyon, IPNL, IN2P3-CNRS, F-69622 Villeurbanne Cedex, France

${ }^{26}$ Universidad Complutense, Avda. Complutense s/n, E-28040 Madrid, Spain

${ }^{27}$ Univ. d'Aix - Marseille II - CPP, IN2P3-CNRS, F-13288 Marseille Cedex 09, France

${ }^{28}$ Dipartimento di Fisica, Università di Milano and INFN, Via Celoria 16, I-20133 Milan, Italy

${ }^{29}$ Niels Bohr Institute, Blegdamsvej 17, DK-2100 Copenhagen 0, Denmark

${ }^{30}$ NC, Nuclear Centre of MFF, Charles University, Areal MFF, V Holesovickach 2, 180 00, Praha 8, Czech Republic

${ }^{31}$ NIKHEF, Postbus 41882, NL-1009 DB Amsterdam, The Netherlands

${ }^{32}$ National Technical University, Physics Department, Zografou Campus, GR-15773 Athens, Greece

${ }^{33}$ Physics Department, University of Oslo, Blindern, N-1000 Oslo 3, Norway

${ }^{34}$ Dpto. Fisica, Univ. Oviedo, Avda. Calvo Sotelo, S/N-33007 Oviedo, Spain, (CICYT-AEN96-1681)

${ }^{35}$ Department of Physics, University of Oxford, Keble Road, Oxford OX1 3RH, UK

${ }^{36}$ Dipartimento di Fisica, Università di Padova and INFN, Via Marzolo 8, I-35131 Padua, Italy

${ }^{37}$ Rutherford Appleton Laboratory, Chilton, Didcot OX11 OQX, UK

${ }^{38}$ Dipartimento di Fisica, Università di Roma II and INFN, Tor Vergata, I-00173 Rome, Italy

${ }^{39} \mathrm{CEA}$, DAPNIA/Service de Physique des Particules, CE-Saclay, F-91191 Gif-sur-Yvette Cedex, France

${ }^{40}$ Istituto Superiore di Sanità, Ist. Naz. di Fisica Nucl. (INFN), Viale Regina Elena 299, I-00161 Rome, Italy

${ }^{41}$ Instituto de Fisica de Cantabria (CSIC-UC), Avda. los Castros, S/N-39006 Santander, Spain, (CICYT-AEN96-1681)

${ }^{42}$ Inst. for High Energy Physics, Serpukov P.O. Box 35, Protvino, (Moscow Region), Russian Federation

${ }^{43}$ Department of Astroparticle Physics, School of Environmental Sciences, Nova Gorica, and J. Stefan Institute, Ljubljana, Slovenia

${ }^{44}$ Fysikum, Stockholm University, Box 6730, S-113 85 Stockholm, Sweden

${ }^{45}$ Dipartimento di Fisica Sperimentale, Università di Torino and INFN, Via P. Giuria 1, I-10125 Turin, Italy

${ }^{46}$ Dipartimento di Fisica, Università di Trieste and INFN, Via A. Valerio 2, I-34127 Trieste, Italy and Istituto di Fisica, Università di Udine, I-33100 Udine, Italy

${ }^{47}$ Univ. Federal do Rio de Janeiro, C.P. 68528 Cidade Univ., Ilha do Fundão BR-21945-970 Rio de Janeiro, Brazil

${ }^{48}$ Department of Radiation Sciences, University of Uppsala, P.O. Box 535, S-751 21 Uppsala, Sweden

${ }^{49}$ IFIC, Valencia-CSIC, and D.F.A.M.N., U. de Valencia, Avda. Dr. Moliner 50, E-46100 Burjassot (Valencia), Spain

${ }^{50}$ Institut für Hochenergiephysik, Österr. Akad. d. Wissensch., Nikolsdorfergasse 18, A-1050 Vienna, Austria

${ }^{51}$ Inst. Nuclear Studies and University of Warsaw, Ul. Hoza 69, PL-00681 Warsaw, Poland

${ }^{52}$ Fachbereich Physik, University of Wuppertal, Postfach 100 127, D-42097 Wuppertal, Germany

${ }^{53}$ On leave of absence from IHEP Serpukhov
} 


\section{Introduction}

In Quantum Chromodynamics (QCD), quarks and gluons carry different colour charges and therefore have different probabilities of emitting additional gluons. Hence, jets originating from the fragmentation of energetic quarks and gluons are expected to show differences in their particle multiplicity, energy spectrum, and angular distribution.

The LEP detectors can select gluon jets in $b \bar{b} g$ events by tagging the $b$ quarks, using selections based on the presence of particles with large impact parameters. This technique has allowed conclusive measurements of the above differences in behaviour of quark and gluon jets from LEP data (see for example Refs. [1-3], and Ref. [4] for recent reviews). From first order QCD and in the asymptotic limit, the hadron multiplicity is expected to be higher in gluon jets than in quark jets by the factor $C_{A} / C_{F}=9 / 4$, but including higher order terms and energy conservation leads to lower values [5]. The experimental results also point to lower values, $\simeq 1.5$ or below and typically found to be about 1.25 , that depend on how the jets are defined [1-3] and increase with energy [3].

No systematic comparisons of identified particle yields in quark and gluon jets have yet been published, although a higher $\eta^{0}$ rate in gluon jets has been reported recently [6]. The DELPHI detector at LEP, equipped with powerful systems for particle identification $[7,8]$, can provide information on the spectra of identified particles in quark and gluon jets, thus testing the predictions of QCD based models in finer detail, and possibly providing hints for better separating quark jets from gluon jets.

The study of the spectra of identified particles $\left(K^{+}, K^{0} \text {, proton and } \Lambda\right)^{\dagger}$ in quark jets and gluon jets from selected symmetric 3 -jet topologies is the subject of this paper. The paper is organized as follows. Section 2 describes the hadronic event selection, the quark/gluon separation, and the particle identification. The experimental results are presented and discussed in comparison with the predictions of models in Section 3. Finally the conclusions are presented in Section 4.

\section{Experimental Technique and Event Sample}

The DELPHI detector and its performance are described in $[7,8]$.

\section{$2.1 \quad$ Event selections}

A sample of hadronic events was selected by requiring 5 or more charged particles with a combined energy of at least $12 \%$ of the beam energy. A charged particle was required to have a momentum, $p$, of more than $400 \mathrm{MeV} / c$, a track length of at least 30 $\mathrm{cm}$, and a polar angle to the beam direction, $\theta$, between $20^{\circ}$ and $160^{\circ}$ [8]. The selection efficiency was about $95 \%$ for hadronic $Z$ decays. The data sample passing the hadronic criteria contained $1,393,000$ events with a small contamination $(<0.7 \%)$ arising from $\tau^{+} \tau^{-}$pairs, beam-gas scattering and $\gamma \gamma$ interactions [8]. Only the data collected during 1994 were used in this analysis, in order to profit from the full operation of the main particle identification detector, the RICH [8], and from the Vertex Detector of DELPHI.

The influence of the detector on the analysis was studied with the full DELPHI simulation program, DELSIM [9]. Events generated with the JETSET 7.3 Parton Shower (PS) model [10], with parameters tuned by DELPHI [11], were passed through DELSIM and processed with the same reconstruction and analysis programs as the real data.

\footnotetext{
${ }^{\dagger}$ Here and in the following, unless otherwise stated, antiparticles are included as well.
} 
Simulations based on JETSET 7.4 PS and HERWIG 5.8 [12] with parameters tuned by DELPHI [11] were also used.

Three-jet events were selected by means of the $k_{\perp}$ (or Durham) jet algorithm [13]. In this algorithm, a jet resolution variable $y_{i j}$ is defined for all pairs of particles:

$$
y_{i j}=\frac{2 \cdot \min \left(E_{i}^{2}, E_{j}^{2}\right) \cdot\left(1-\cos \alpha_{i j}\right)}{E_{v i s}^{2}}
$$

where $\alpha_{i j}$ is the angle between the two particles, $E_{i}\left(E_{j}\right)$ is the particle energy (obtained in our case from the particle momenta by assuming the pion mass for the charged and massless neutrals except in the case of a $V^{0}$, for which the mass of the $V^{0}$ itself was used), and $E_{v i s}$ is the sum of all particle energies observed in the event. The particle pair with the smallest $y_{i j}$, if its $y_{i j}$ is smaller than a cut-off value $y_{c u t}$, is replaced by a pseudoparticle with four-momentum equal to the sum of the four-momenta of particles $i$ and $j$. This procedure is repeated until all $y_{i j}$ are greater than $y_{c u t}$. At the end of the procedure, the remaining (pseudo)particles are the jets. The value used for the cut-off, $y_{\text {cut }}=0.015$, was optimized using the JETSET 7.3 PS model, by maximizing the statistics available and the quark/gluon purity attained for the three-jet event samples [3], thus allowing a reliable comparison with perturbative QCD. Both charged and neutral particles were used in the jet reconstruction algorithm. The number of 3-jet events selected was 359084 .

Two samples of 3 -jet events with different geometries were used:

- "Y events", two-fold symmetrical events with each of the two angles $\theta_{2}$ and $\theta_{3}$ (see Fig. 1) in the interval between $135^{\circ}$ and $165^{\circ}$ : only the two closest jets (jets 2 and 3 in Fig. 1) were used in the analysis, and the condition $\left|\theta_{2}-\theta_{3}\right| \leq 15^{\circ}$ was imposed in order to limit the energy difference between them.

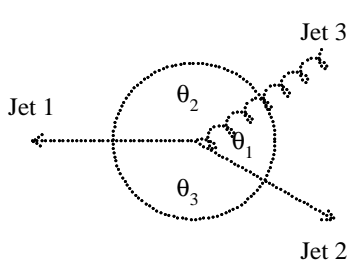

Y events

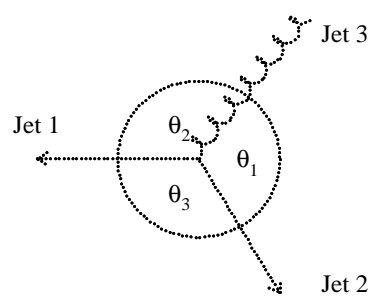

Mercedes events

Figure 1: Geometry of $Y$ and Mercedes type events.

- "Mercedes events", three-fold symmetrical events with each of the three angles $\theta_{1}$, $\theta_{2}$ and $\theta_{3}$ in Fig. 1 in the interval between $100^{\circ}$ and $140^{\circ}$. All three jets were used in the analysis.

In both cases, all three jets were required to have polar angles to the beam direction between $30^{\circ}$ and $150^{\circ}$, and the planarity condition $\theta_{1}+\theta_{2}+\theta_{3} \geq 355^{\circ}$ was imposed.

The advantage of using Mercedes and $Y$ events in this way is that the gluon and at least one quark jet have about the same energy, thus removing phase space effects. The disadvantages are the limited range of parton energies spanned and the severely limited statistics. The numbers of 3 -jet events in these Mercedes and Y samples were equal to 9805 and 59166 respectively. 
The energies of the jets were calculated from the jet directions and the angles between them. Assuming massless kinematics, the jet energies can be expressed as:

$$
p_{j}^{\text {calc }}=E_{j}^{\text {calc }}=\frac{\sin \theta_{j}}{\sin \theta_{1}+\sin \theta_{2}+\sin \theta_{3}} \sqrt{s}, \quad j=1,2,3
$$

where $\theta_{j}$ is the inter-jet angle as defined in Fig. 1. Studies using a full simulation of the DELPHI detector showed [3] that, for the whole available range of jet energies, $E_{j}^{\text {calc }}$ gives a better representation of the true jet energy than the reconstructed (or visible) jet energy does. The use of expression (2) corrects for the energy shift towards low values due to particle loss, and improves the energy resolution from about $\pm 2.5 \mathrm{GeV}$ to about $\pm 1 \mathrm{GeV}$.

\subsection{Quark/gluon Separation}

The probability of producing $b$-quark pairs inside gluon jets is expected to be small [14]. Gluon jets can therefore be collected from a sample of reconstructed $b \bar{b} g$ three-jet events by directly identifying the two quark jets as originating from $b$ quarks. The experimental techniques employed in the present analysis detect $b$-jets efficiently, enable reasonably high gluon jet purities to be attained, and thus allow the study of a sample of gluon jets containing only a small background.

The $b$-jet tagging was done after requiring the probability $P_{E}[8,15]$, for the hypothesis that none of the charged particles with positive impact parameter in the event came from a secondary vertex, to be smaller than $2 \times 10^{-2}$. The $b \bar{b}$ purity attained was about $71.2 \%$ and $69.5 \%$ in the Mercedes and $\mathrm{Y}$ samples, respectively.

In the Mercedes events, the gluon candidate was then selected as the jet with the largest $P_{J}$, provided it had $P_{J}$ above 0.1 and the two other jets had $P_{J}$ below 0.1 , where $P_{J}$ is calculated like $P_{E}$ but using only the charged particles in a given jet.

In $\mathrm{Y}$ events, the gluon candidate was selected as the jet with largest $P_{J}$, provided it was greater than 0.1. If this was the most isolated jet (jet 1 in Fig. 1 left), the event was discarded. It was required in addition that the nearest jet had $P_{J} \leq 0.1$.

After $b$ tagging, the numbers of 3 -jet events in the Mercedes and $Y$ samples were equal to 1090 and 7017 respectively.

The average and root-mean-square spread of the energies of the jets selected as $b$ and gluon jets are indicated in table 1, for both the Mercedes and Y events.

\begin{tabular}{|l||c|c|}
\hline Class & gluon & $b$ \\
\hline \hline \multicolumn{3}{|c|}{ Mercedes Events } \\
\hline Average energy (GeV) & 29.5 & 30.9 \\
RMS spread (GeV) & 3.4 & 3.2 \\
\hline \hline \multicolumn{3}{|c|}{ Y Events } \\
\hline Average energy (GeV) & 23.5 & 25.0 \\
RMS spread (GeV) & 3.3 & 3.2 \\
\hline
\end{tabular}

Table 1: Averages and root-mean-square spreads of the energies of the $b$ and gluon jets selected in the Mercedes and $Y$ events.

Three classes of jets were considered in the following analysis:

- a $g$-enriched class, containing the gluon candidates selected as just described; 
- a $b$-enriched class, containing the two jets not selected for the $g$-enriched class in the Mercedes sample, and the non-gluon jet among jet 2 and jet 3 in the $Y$ sample;

- a reference class, containing all the jets in the Mercedes events, and all jets 2 and 3 in the $\mathrm{Y}$ events, before $b$ tagging.

The compositions of these samples were calculated using events generated with the JETSET 7.3 PS model, which were subsequently passed through DELSIM, to simulate detector effects, and the jets were then reconstructed. In each event, the generated particles were clustered into the same number of jets as had been reconstructed (three in the selected samples). Two different methods were then used to assign the reconstructed jets to the generated jets :

- Generated heavy hadrons were assigned to the generated jets, and the reconstructed jet which had the largest angle to the generated heavy hadron jets was assumed to be the gluon induced jet.

- Partons were clustered into three jets, and the reconstructed jets were associated to the parton jet closest in angle.

The two methods were in good agreement.

The calculated compositions of the three jet classes determined using these procedures are summarized in table 2 for Mercedes and Y events. The gluon fractions in the reference samples are easily understood, since by symmetrization we expect $1 / 3$ of the jets in Mercedes events to be gluon, and nearly $1 / 2$ in $\mathrm{Y}$ events.

\begin{tabular}{|l||c|c|c|}
\hline Class & $g$ & $b$ & $u d s c$ \\
\hline \hline \multicolumn{4}{|c|}{ Mercedes Events } \\
\hline g-enriched & 0.828 & 0.069 & 0.102 \\
b-enriched & 0.076 & 0.774 & 0.148 \\
Reference & 0.334 & 0.143 & 0.521 \\
\hline \hline \multicolumn{4}{|c|}{ Y Events } \\
\hline g-enriched & 0.837 & 0.068 & 0.093 \\
b-enriched & 0.104 & 0.720 & 0.174 \\
Reference & 0.462 & 0.110 & 0.426 \\
\hline
\end{tabular}

Table 2: Fractional compositions of the three jet classes for Mercedes and $Y$ events.

\subsection{Identification of Final State Particles}

The $K^{+}$and protons were tagged using the Cherenkov angle measurement in the RICH detector and the ionization energy loss $(d E / d x)$ in the TPC. The $d E / d x$ information was used to identify $K^{+}$for momenta below $0.7 \mathrm{GeV} / c$ and protons below $0.9 \mathrm{GeV} / c$, where no RICH information is available. At higher momenta, due to the better resolution and better separation between the expectation curves, the tagging performance of the RICH is superior, so the tagging was performed mainly using the RICH.

The RICH analysis was restricted to the barrel RICH region $\left(41^{\circ} \leq \theta \leq 139^{\circ}\right)$. The RICH hadron identification was based on three standard software packages, 'RICFIX', 'RIBMEAN' and 'NEWTAG' [16]. RICFIX corrects the real and simulated RICH data 
for detector related effects (such as slight fluctuations in pressures and refractive indices, background arising from photon feedback, cross-talk between readout strips and wires, $\delta$-rays, track ionization photoelectrons, etc.) in order to optimise the performance and to ensure good agreement between data and simulation. RIBMEAN then estimates the Cherenkov angles in the liquid and gas radiators by applying a clustering algorithm to the detected Cherenkov photons, and simultaneously assigns a quality flag to each charged particle (track) passing through the RICH. Finally NEWTAG performs the $\pi, K^{+}$and proton tagging. Basically, a particle is tagged if its measured Cherenkov angle is within 2.5 standard deviations of the prediction of the given mass hypothesis and at least 1 (loose tag), 2 (standard tag) or 3 (tight tag) standard deviations from the nearest neighbour hypothesis. The exact cuts depend on the particle type and momentum, in order to take into account the varying separation quality of the detectors involved.

In the momentum range below $0.9 \mathrm{GeV} / c$, the clearly separated bands corresponding to electron, pion, kaon, and proton in the plot of $d E / d x$ versus momentum were used for identification (muons can not be distinguished from pions). Detailed calibration was performed as described in [17].

The efficiency averaged over the momentum spectrum was estimated from the full detector simulation to be $56 \%(46 \%)$ with a purity of $75 \%(92 \%)$ for $K^{+}$(proton), in the sample of events selected for this analysis. Fig. 2 shows the efficiency and contamination as a function of momentum for the $K^{+}$and protons selected.

The $K_{S}^{0}$ and $\Lambda$ candidates were detected by their decay in flight into $\pi^{+} \pi^{-}$and $p \pi^{-}$ respectively. Candidates were found by considering all pairs of oppositely charged particles. The vertex defined by each such pair was determined by minimising the $\chi^{2}$ for the hypothesis of a common vertex, and the track parameters were refitted to the common vertex. The selection criteria were the "tight" ones described in [8]. The average detection efficiency of this procedure is about $36 \%$ for $K_{S}^{0} \rightarrow \pi^{+} \pi^{-}$and about $28 \%$ for $\Lambda$ $\rightarrow p \pi^{-}$in multihadronic events (Fig. 2). The backgrounds under the invariant mass peaks were subtracted, separately for each momentum bin, by linear interpolation between two side-bands in invariant mass: these were the regions between 0.40 and $0.45 \mathrm{GeV} / \mathrm{c}^{2}$ and between 0.55 and $0.60 \mathrm{GeV} / \mathrm{c}^{2}$ for the $K_{S}^{0}$, and the regions between 1.08 and $1.10 \mathrm{GeV} / \mathrm{c}^{2}$ and between 1.14 and $1.18 \mathrm{GeV} / \mathrm{c}^{2}$ for the $\Lambda$.

\section{Analysis and Results}

The production of identified particles in the final state was studied in four momentum bins for $K^{0}$ and $\Lambda$, and in six momentum bins for charged kaons and protons, as indicated in table 3 .

\begin{tabular}{|c|c|c|c|c|c|c|}
\hline Particle & \multicolumn{6}{|c|}{$K^{0}, \Lambda$} \\
\hline $\begin{array}{l}\text { Momentum bins } \\
(\mathrm{GeV} / \mathrm{c})\end{array}$ & $\begin{array}{c}\operatorname{bin} 1 \\
0.5-2.0\end{array}$ & \multicolumn{2}{|c|}{$\begin{array}{c}\text { bin } 2 \\
2.0-5.0\end{array}$} & \multicolumn{2}{|c|}{$\begin{array}{c}\text { bin } 3 \\
5.0-10.0\end{array}$} & $\begin{array}{c}\text { bin } 4 \\
10.0-25.0\end{array}$ \\
\hline Particle & \multicolumn{6}{|c|}{$K^{+}$, proton } \\
\hline $\begin{array}{l}\text { Momentum bins } \\
(\mathrm{GeV} / \mathrm{c})\end{array}$ & $\begin{array}{l}\operatorname{bin} 1 \\
0.3-0.5\end{array}$ & $\begin{array}{l}\text { bin } 2 \\
0.5-0.9\end{array}$ & $\begin{array}{l}\text { bin } 3 \\
0.9-2.3\end{array}$ & $\begin{array}{c}\operatorname{bin} 4 \\
2.3-4.5\end{array}$ & $\begin{array}{c}\text { bin } 5 \\
4.5-9.0\end{array}$ & $\begin{array}{c}\operatorname{bin} 6 \\
9.0-25.0\end{array}$ \\
\hline
\end{tabular}

Table 3: Momentum bins used for the study of identified particles. 

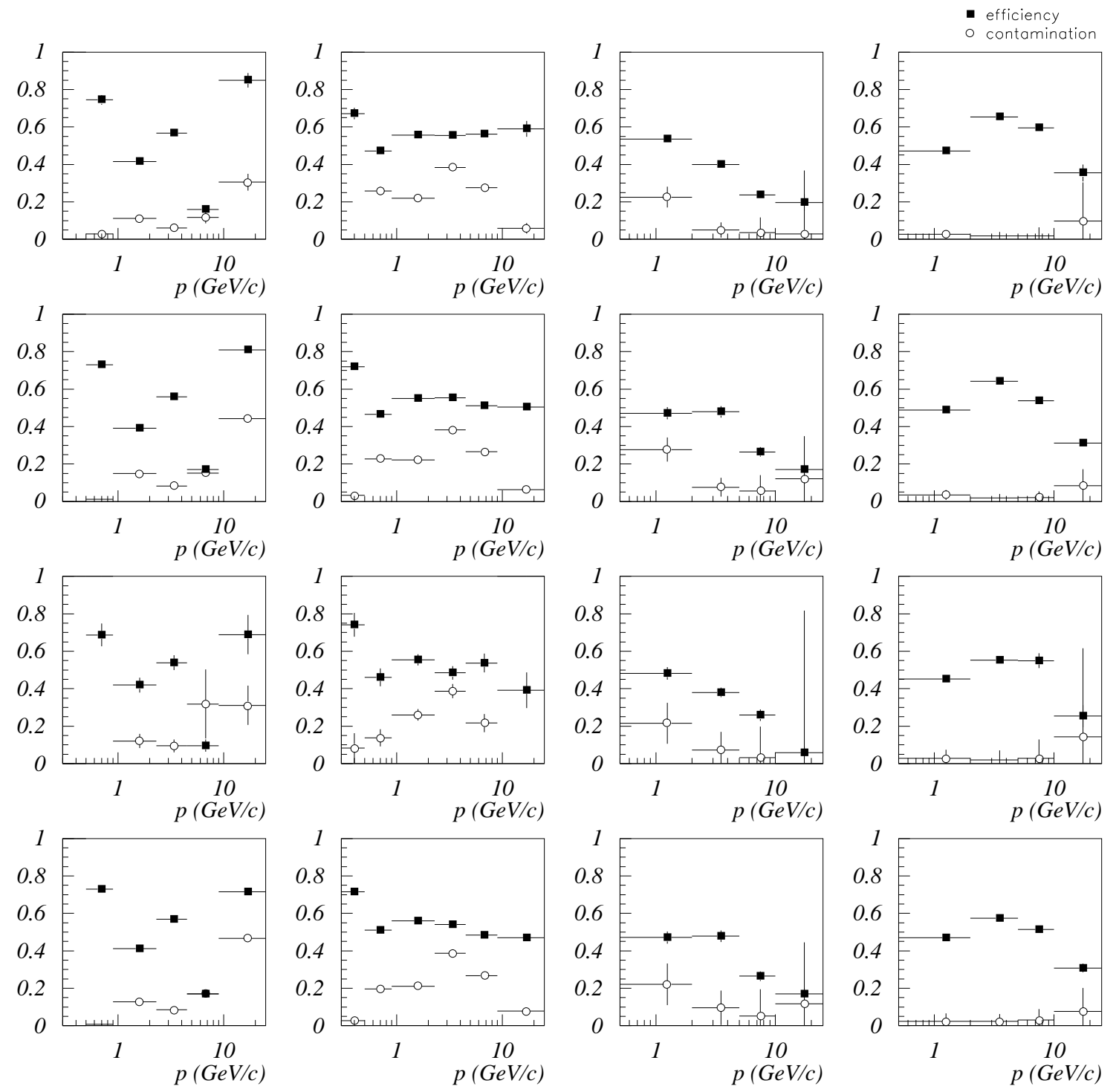

Figure 2: Efficiency (squares) and contamination (open circles) as a function of momentum for $Y$ events and Mercedes events for different types of particle: the histograms in the 1st, 2nd, 3rd and 4th columns correspond to particle types $p, K^{+}, \Lambda$ and $K^{0}$ respectively. The 1st and 2 nd rows of histograms refer to $Y$-type events, the 1 st row for gluon jets and the 2 nd row for quark jets. The 3rd row corresponds to gluon jets in Mercedes type events and the 4 th row to quarks jets in Mercedes type events. 
The ratios of the momentum distributions, not yet corrected for the contamination of the different jet classes or for the reconstruction efficiency of the particles in those jets, are shown in Fig. 3 for the $g$-enriched class relative to the reference class, together with the same ratios for charged particles. The simulation sample used consisted of about 4.6 $\cdot 10^{6}$ hadronic $Z$ decays generated with the JETSET 7.3 PS model, with JETSET 7.4 PS and with HERWIG 5.8. The spectra of identified particles in the class enriched in gluon jets appear to be softer than the corresponding spectra in the reference class.

The effects of the contaminations in the jet classes were unfolded by applying an algebraic correction method to the momentum distributions. The method uses the compositions of the classes of jets in table 2 as the only input from simulation. If $M_{g-e n r i c h e d}\left(m_{i}\right)$, $M_{b-e n r i c h e d}\left(m_{i}\right)$, and $M_{\text {reference }}\left(m_{i}\right)$ respectively represent the momentum distributions constructed from the $g$-enriched class, the $b$-enriched class, and the reference class, where $m_{i}$ is the content of bin $i$, then

$$
M_{j}\left(m_{i}\right)=P_{g}(j) \cdot G\left(m_{i}\right)+P_{b}(j) \cdot B\left(m_{i}\right)+P_{q}(j) \cdot Q\left(m_{i}\right)
$$

where $G\left(m_{i}\right), B\left(m_{i}\right)$ and $Q\left(m_{i}\right)$ are the distributions for pure $g$, pure $b$ and pure $q=u d s c$ jets respectively, with $P_{g}(j), P_{b}(j)$, and $P_{q}(j)$ being the fractions of the jets in class $j$ ( $j=g$-enriched, $b$-enriched and reference) which are pure $g, b$, and $q=u d s c$ respectively, as given in table 2 . These equations can be solved to extract the pure $g, b$, and $q=u d s c$ contributions.

Only two pure classes were extracted in the present analysis: the class of pure gluons and a pure quark class including all quarks $(q=u d s c b)$ in the proportions predicted by the standard model for $Z$ decay into quarks. This $q=u d s c b$ class was obtained from the compositions of $u d s c$ and $b$ quarks in the enriched classes and the reference class of table 2, neglecting the $c$-enrichment in the $b$-enriched class: this was however accounted for later, in the systematic uncertainties.

The reconstruction efficiencies were determined, using the JETSET 7.3 PS model, by comparing the momentum distributions of the identified particles in the two pure classes of jets extracted from the simulated events with those extracted from the generated ones.

The ratios obtained, after unfolding the contamination of the jet classes and correcting for the reconstruction efficiencies of the particles in the pure jet classes, are shown in Fig. 4, together with the corresponding ratios for charged particles.

The ratio of the charged multiplicities in gluon jets relative to quark jets averaged over the momentum spectrum was found to be $r_{c h}=1.22 \pm 0.01$ and $r_{c h}=1.30 \pm 0.03$ for $\mathrm{Y}$ and Mercedes events respectively, consistent with the dependence of this ratio on the jet energy observed previously [3].

Normalized ratios, $R_{X}^{\prime}(p)$, were then defined by :

$$
R_{X}^{\prime}(p)=\frac{r_{X}(p)}{r_{c h}(p)},
$$

where $r_{X}(p)$ is the fully corrected ratio of the multiplicity measured in gluon jets relative to quark jets as a function of the momentum $p$ for the identified particle $X\left(X=K^{0}, \Lambda\right.$, $K^{+}$, proton), and $r_{c h}(p)$ is the corresponding ratio for all charged particles.

The normalized ratios shown in Fig. 5 are computed from the ratios in Fig. 4 . The normalized ratios integrated over the momentum spectrum, $R_{X}^{\prime}$, are listed in table 4 , and compared with the predictions from the simulation.

The systematic uncertainties on these ratios were estimated by summing in quadrature the following sources. 


\begin{tabular}{|l||c|c|c|c|}
\hline$R_{X}^{\prime}$ & Measured & JETSET 7.3 PS & JETSET 7.4 PS & HERWIG 5.8 \\
\hline \hline \multicolumn{4}{|c|}{ Y Events } \\
\hline$R_{p}^{\prime}$ & $1.12 \pm 0.11 \pm 0.04$ & $1.36 \pm 0.03$ & $1.53 \pm 0.05$ & $0.94 \pm 0.02$ \\
$R_{K^{+}}^{\prime}$ & $0.93 \pm 0.04 \pm 0.02$ & $0.83 \pm 0.01$ & $0.84 \pm 0.01$ & $0.70 \pm 0.01$ \\
$R_{\Lambda}^{\prime}$ & $1.40 \pm 0.30 \pm 0.23$ & $1.40 \pm 0.06$ & $1.53 \pm 0.07$ & $1.02 \pm 0.03$ \\
$R_{K^{0}}^{\prime}$ & $1.13 \pm 0.09 \pm 0.13$ & $0.94 \pm 0.02$ & $0.98 \pm 0.02$ & $0.93 \pm 0.01$ \\
\hline \hline \multicolumn{5}{|c|}{ Mercedes Events } \\
\hline$R_{p}^{\prime}$ & $1.25 \pm 0.22 \pm 0.05$ & $1.43 \pm 0.05$ & $1.35 \pm 0.06$ & $1.07 \pm 0.04$ \\
$R_{K^{+}}^{\prime}$ & $0.92 \pm 0.09 \pm 0.03$ & $0.82 \pm 0.02$ & $0.84 \pm 0.02$ & $0.68 \pm 0.02$ \\
\hline
\end{tabular}

Table 4: Normalized ratios $R_{X}^{\prime}$ (see text) integrated over the momentum spectrum and compared to the predictions from the Monte Carlo simulations. The first error quoted is statistical, the second is systematic. The breakdown of the systematic error is given in Table 5.

1. An overall uncertainty of $\pm 5 \%$ was used for the $K^{+}$and proton identification efficiency and purity, deduced from the simulation by comparing the results from loose, standard and tight tagging for different track quality samples. For $K^{0}$ and $\Lambda$, this systematic uncertainty was taken as $\pm 15 \%$.

2. The uncertainties on the flavour compositions in table 2 were found by assuming that all the $c$ quark jets in the $g$-enriched and $b$-enriched classes were in fact $b$ quark jets. In addition, the gluon jet purity was varied by $5 \%$ in the $\mathrm{Y}$ and Mercedes samples. The larger of the two variations was taken as the estimator of the systematic effects.

3 . The effect of neglecting the $c$-enrichment in the $b$-enriched class when unfolding the effect of the contamination of the jet classes was estimated from the effects of considering all $c$ quarks as $b$ 's, and all uds quarks as b's. The half distance between the two results was taken as a conservative estimate of this systematic uncertainty.

The effects of these sources of systematic uncertainty on the normalized ratios are summarized in table 5 .

It can be seen in Table 4 that all the $R_{X}^{\prime}$ values are consistent with unity, ie the ratios of the average multiplicities in $g$ jets and $q$ jets for all identified particles are consistent with the corresponding ratios for charged particles. The value of $R_{p}^{\prime}$ in Y events is about 1.5 standard deviations higher than predicted by HERWIG 5.8, and 2 to 3 standard deviations lower than predicted by JETSET. The value of $R_{K^{+}}^{\prime}$ is higher than predicted by HERWIG 5.8 by about 5 standard deviations in $Y$ events, and by about 2.5 standard deviations in Mercedes events.

\section{Conclusions}

Based on a sample of about 1.4 million hadronic $Z$ decays collected by the DELPHI detector at LEP, the production spectra of identified particles in jets initiated by gluons and jets initiated by quarks, were analysed in order to search for possible differences between gluons and quarks jets. 


\begin{tabular}{|l||c|c|c|c|}
\hline$R_{X}^{\prime}$ & Source 1 & Source 2 & Source 3 & Total \\
\hline \hline \multicolumn{5}{|c|}{ Y Events } \\
\hline$R_{p}^{\prime}$ & 0.011 & 0.010 & 0.038 & 0.041 \\
$R_{K^{+}}^{\prime}$ & 0.014 & 0.001 & 0.014 & 0.020 \\
$R_{\Lambda}^{\prime}$ & 0.139 & 0.130 & 0.123 & 0.227 \\
$R_{K^{0}}^{\prime}$ & 0.112 & 0.074 & 0.008 & 0.134 \\
\hline \hline \multicolumn{5}{|c|}{ Mercedes Events } \\
\hline$R_{p}^{\prime}$ & 0.013 & 0.003 & 0.050 & 0.052 \\
$R_{K^{+}}^{\prime}$ & 0.014 & 0.020 & 0.015 & 0.029 \\
\hline
\end{tabular}

Table 5: Systematic uncertainties on the integrated normalized ratios $R_{X}^{\prime}$ given in Table 4.

As observed for inclusive charged particles, the production spectrum of identified particles was found to be softer in gluon jets compared to quark jets, with a higher total multiplicity.

For all identified particles, the ratio of the average multiplicity in $g$ jets with respect to $q$ jets was found to be consistent with the same ratio measured for charged particles. However, for protons, the ratio normalised to the ratio for charged particles in $\mathrm{Y}$ events is about 1.5 standard deviations higher than predicted by HERWIG 5.8, and 2 to 3 standard deviations lower than predicted by JETSET. For charged kaons, the normalised ratio is higher than predicted by HERWIG 5.8 by about 5 standard deviations in Y events, and by about 2.5 standard deviations in Mercedes events.

\section{Acknowledgements}

We are greatly indebted to our technical collaborators and to the funding agencies for their support in building and operating the DELPHI detector, and to the members of the CERN-SL Division for the excellent performance of the LEP collider. 


\section{References}

[1] OPAL Coll., G. Alexander et al., Phys. Lett. B265 (1991) 462;

OPAL Coll., P.D. Acton et al., Z. Phys. C58 (1993) 387;

OPAL Coll., R. Akers et al., Z. Phys. C68 (1995) 179;

OPAL Coll., G. Alexander et al., Z. Phys. C69 (1996) 543;

OPAL Coll., G. Alexander et al., CERN-PPE/96-116, subm. to Phys. Lett. B.

[2] ALEPH Coll., D. Buskulic et al., Phys. Lett. B346 (1995) 389;

ALEPH Coll., D. Buskulic et al., Phys. Lett. B384 (1996) 353.

[3] DELPHI Coll., P. Abreu et al., Z. Phys. C70 (1996) 179 (and references therein).

[4] J. Fuster and S. Marti, "Charged Particle Production in the Fragmentation of Quark and Gluon Jets", Proc. of the EPS-HEP Conference (Bruxelles 1995), p. 319;

I.G. Knowles et al., Physics at LEPD, Vol. 2, CERN 96-01, eds. G. Altarelli and F. Zwirner.

[5] S.J. Brodsky, J. Gunion, Phys. Rev. Lett. 37 (1976) 402;

K. Konishi, A. Ukawa, G. Veneziano, Phys. Lett. B78 (1978) 343;

A.H. Mueller, Nucl. Phys. B241 (1984) 109;

J.B. Gaffney, A.H. Mueller, Nucl. Phys. B250 (1985) 501;

E.D. Malaza, B.R. Webber, Phys. Lett. B149 (1984) 501;

E.D. Malaza, Z. Phys. C31 (1986) 143;

I.M. Dremin, R.C. Hwa, Phys. Lett. B324 (1994) 477;

I.M. Dremin, V.A. Nechitailo, Mod. Phys. Lett. A9 (1994) 1471.

[6] L3 Coll., M. Acciarri et al., Phys. Lett. B371 (1996) 126.

[7] DELPHI Coll., P. Abreu et al., Nucl. Instr. Meth. A303 (1991) 233.

[8] DELPHI Coll., P. Abreu et al., Nucl. Instr. Meth. A378 (1996) 57.

[9] DELPHI Coll., P. Abreu et al., DELPHI event generation and detector simulation DELPHI 89-67 PROG-142;

DELSIM Reference manual, DELPHI 89-68 PROG-143.

[10] T. Sjöstrand, Comp. Phys. Comm. 82 (1994) 74;

T. Sjöstrand, Comp. Phys. Comm. 39 (1986) 346;

T. Sjöstrand, M. Bengtsson: Comp. Phys. Comm. 43 (1987) 367;

T. Sjöstrand, JETSET 7.3 Program and Manual, CERN-TH 6488/92;

B. Bambah et al., QCD Generators for LEP, CERN-TH 5466/89.

[11] DELPHI Coll., P. Abreu et al., "Tuning and Test of Fragmentation Models Based on Identified Particles and Precision Event Shape Data", CERN-PPE/96-120, to be published in Z. Phys. C.

[12] G. Marchesini et al., Comp. Phys. Comm. 67 (1992) 465.

[13] S. Catani et al., Phys. Lett. B269 (1991) 432.

[14] M.L. Mangano, P. Nason, Phys. Lett. B285 (1992) 160;

M.H. Seymour, Z. Phys. C63 (1994) 99;

M.H. Seymour, Nucl. Phys. B436 (1995) 163.

[15] DELPHI Coll., P. Abreu et al., Z. Phys. C65 (1995) 555.

[16] E. Schyns, "NEWTAG - $\pi, K, p$ tagging for DELPHI RICHes", DELPHI Note 96-103 RICH 89.

[17] J. Dahm, M. Reale and M. Elsing, Calibration of the DELPHI $d E / d x$ for $1991 F$ and 1992D data, DELPHI Note 95-48 TRACK 81. 


\section{g-enriched/Reference}
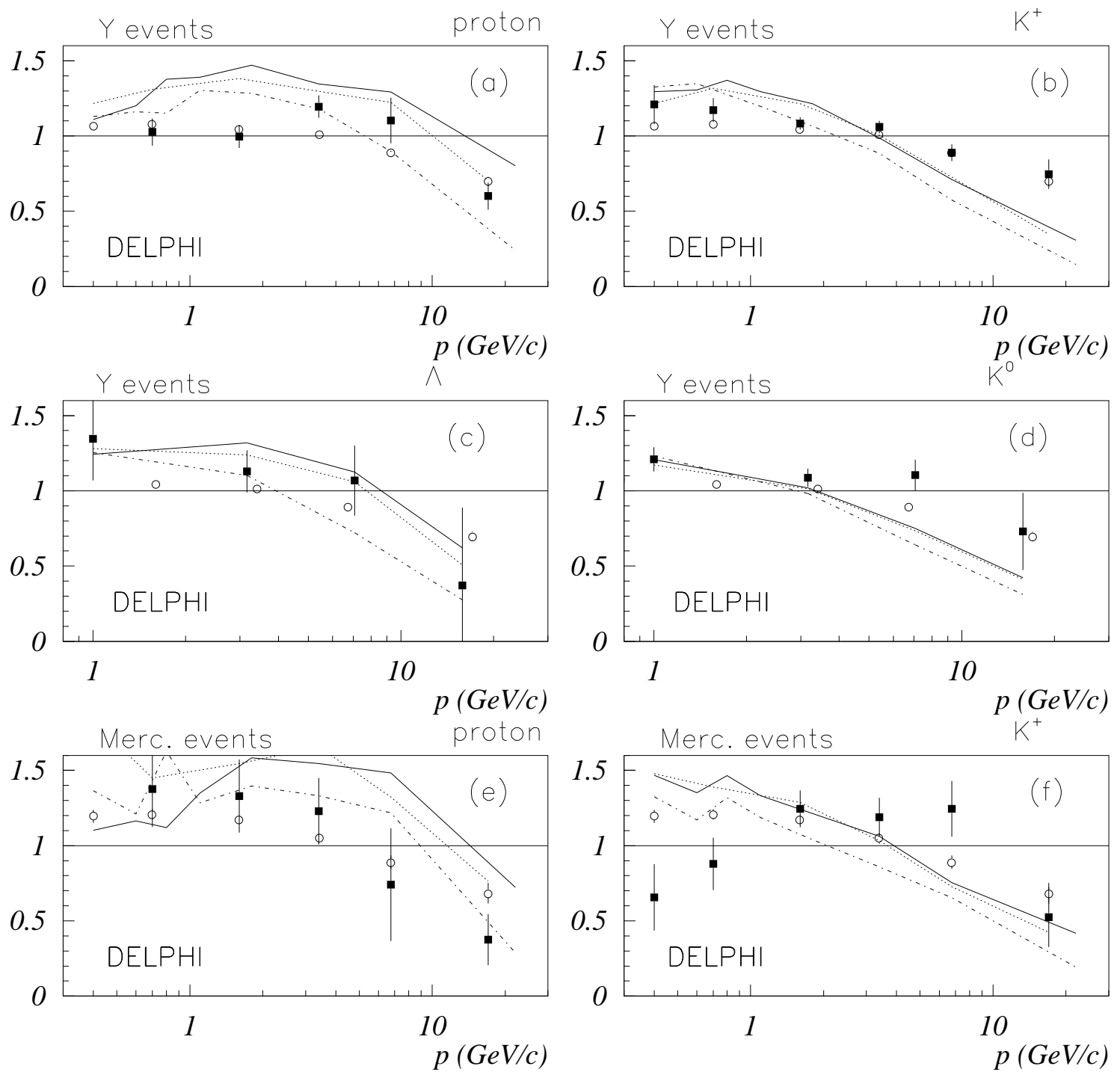

Figure 3: Observed uncorrected ratios of the yields of (a) protons, (b) $K^{+}$, (c) $\Lambda$, and (d) $K^{0}$ in the g-enriched class with respect to the reference class (black squares), for $Y$ events; (e) and ( $f$ ) for Mercedes events correspond to (a) and (b) respectively. The circles represent the observed ratios of the yields of charged particles in the $g$-enriched class with respect to the reference class. The predictions from the JETSET 7.3 PS model are shown as a dotted line, the full line represents the predictions from the JETSET 7.4 $P S$ model, and the dash-dotted line represents the predictions from HERWIG 5.8. 


\section{$\mathbf{g} / \mathbf{q}$}
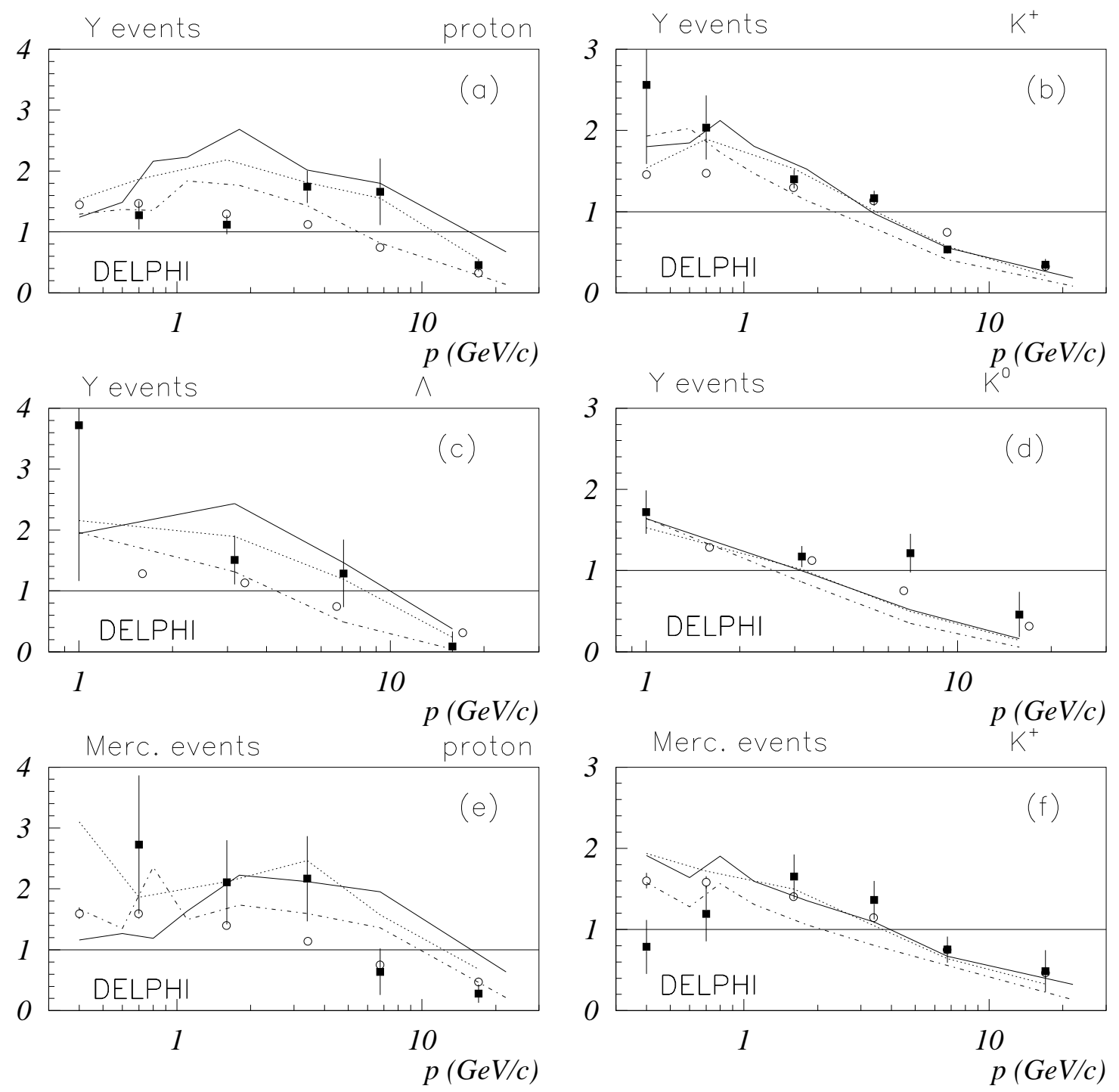

Figure 4: As Fig. 3, but for the fully corrected ratios of yields in gluon jets with respect to quark jets (black squares), and the corresponding model predictions. 


\section{Normalized ratios}
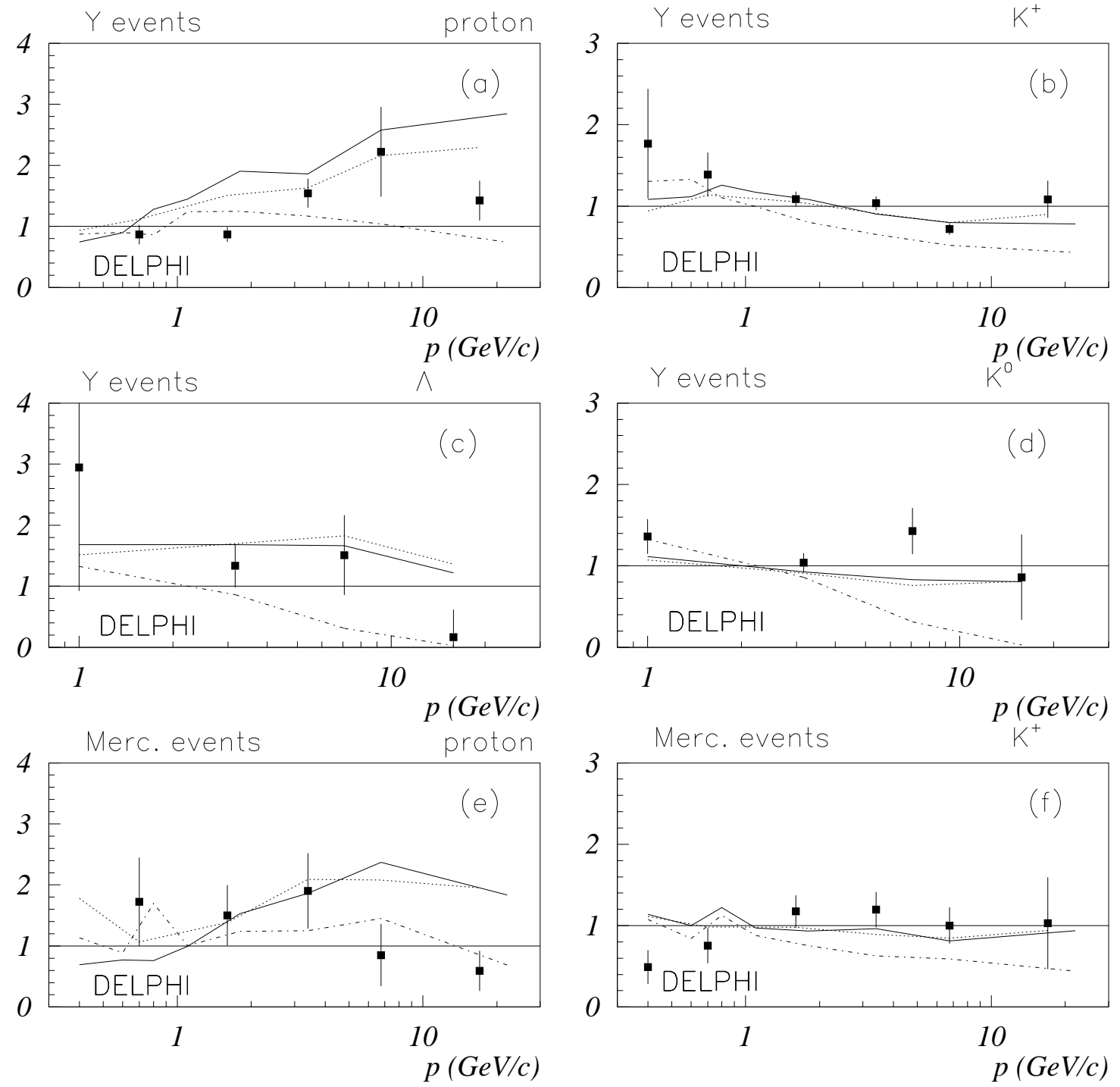

Figure 5: As Fig. 3, but for the normalized ratio, $R_{X}^{\prime}(p)$ defined in the text (black squares), and the corresponding model predictions. 